\title{
TENDENCIAS EN LA ACTIVIDAD Y EN LA INVESTIGACIÓN DE LA CONSTRUCCIÓN
}

\author{
(TENDENCIES IN THE ACTIVITY AND IN THE BUILDING RESEARCH)
}

\author{
F. Aguirre de Yraola, Dr. Arquitecto \\ ICCET/CSIC \\ ESPAÑA
}

\section{RESUMEN}

El propósito del presente artículo es referir la panorámica del futuro de la investigación del sector de la construcción al marco europeo, e informar acerca de los puntos más importantes de las directrices emanadas de la Comunidad Económica Europea que involucran a la Normativa y a la actividad de los Centros de Investigación y Normalización europeos en un próximo futuro.

En especial, se refiere a los organismos en los cuales el ICCET ha entrado a formar parte, tales como la EOTA (European Organisation for Technical Approuval) y el ENBRI (European Network of Building Research Institutes).

\section{SUMMARY}

The purpose of the present paper is to refer the panorama of the future of building research to the European frame, and to inform about the most important items emanated from the European Economic Community which involve the Standardization and the activity of the European organizations in a next future.

Particulary, it refers to the bodies where the Instituto de Ciencias de la Construcción Eduardo Torroja is a new member, like the EOTA (European Organization for Technical Approuval) and the ENBRI (European Network of Building Research Institutes).

\section{TENDENCIAS EN LA ACTIVIDAD Y EN LA INVESTIGACIÓN DE LA CONSTRUCCIÓN}

Últimamente han tenido lugar unos acontecimientos cuya importancia corresponde a la rápida incorporación de nuevos conceptos de diseño, técnicas, procesos constructivos y utilización de los edificios.

El documento elaborado por el Consejo Internacional de la Edificación (C.I.B.), cuyos principales objetivos son la investigación, el estudio y la documentación del sector construcción en el mundo, señala que este sector va evolucionando al ritmo de los desafíos actuales interdependientes de la calidad, la industrialización, el comportamiento y las prestaciones para un mayor confort del usuario. $Y$ que la informatización progresiva de los materiales, producto y tecnologias de base, provocados por el carácter mundial de la economia y el crecimiento de las transferencias tecnológi- cas, provoca un conflicto que no puede ignorarse en el marco de un desarrollo armónico de las tecnologías.

El propósito de las presentes lineas es, sin embargo, referir la panorámica del futuro de la investigación al marco europeo, y dar cuenta de unas actividades que involucran al ICCET en el marco de la Comunidad Europea (Fig. 1).

En primer lugar, a partir del acta única de febrero de 1986, la CEE ha acelerado la legislación comunitaria dedicada a la construcción y, como consecuencia, es importante la publicación de la Directiva de Productos en diciembre de 1988, dedicada a la libre circulación y utilización de materiales y a su calidad.

En esta directiva, como base para las normas armonizadas con otras especificaciones técnicas a nivel europeo, y para la elaboración o concesión de los Docu- 


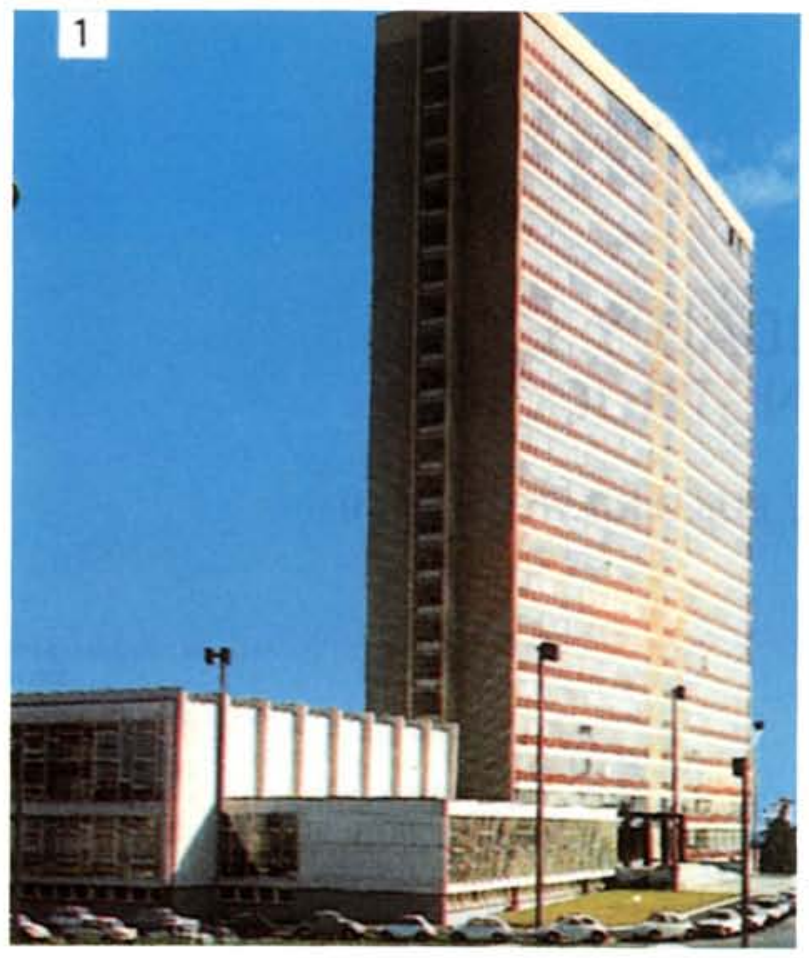

Fig. 1.-Centro Europeo en Luxemburgo.

mentos de Idoneidad Técnica Europeos, se han establecido Documentos Interpretativos con el fin de dar forma correcta a los requisitos esenciales a nivel técnico.

Por otra parte, los principales institutos y centros de investigación dentro del sector de la construcción europeos quedaron, en 1988, agrupados en el organismo internacional ENBRI (European Network Building Research Institutes), uno de cuyos principales objetivos consiste en ayudar al cumplimiento de la directiva "Productos de Construcción" y a la preparación de los Documentos Intepretativos y de la documentación técnica requerida por esa directiva.

En particular, un reducido número de expertos en el tema "Condiciones del interior de los edificios", escogidos entre los institutos miembros del ENBRI, estudiaron la posible estructura de los Documentos interpretativos, con el fin de dar una expresión a los requerimientos esenciales y servir de referencia para el establecimiento de una normativa armonizada y unas guias para la aprobación de la idoneidad técnica europea y para el reconocimiento de las especificaciones técnicas nacionales.

Posteriormente, se creó un grupo titulado "Construction Research Needs in Europe", formado por miembros de los Institutos: CSTB de Francia; BRE del Reino Unido; CSTC de Bélgica; NTO de los Paises Bajos; IfBt de Alemania; SBI de Dinamarca; EOLAS de Irlanda; ICITE de Italia; LNEC de Portugal; y el ICCET de España, que han trabajado en la preparación de unos temas que se han considerado prioritarios para el futuro próximo de la investigación, auspiciado por la CEE.

Esta colaboración entre Centros, fruto de repetidas reuniones en Paris y Bruselas, y del trabajo realizado en cada Instituto por equipos especializados, han culminado con la celebración de un Simposio, a finales de octubre, en Luxemburgo, presidido por el Dr. Courtney, Director del British Research Establishment del Reino Unido y Presidente del ENBRI.

Este Simposio se desarrolló según una estructuración en cinco secciones que correspondieron a los temas:

- Desarrollo de materiales de construcción y su empleo.

- Impacto de la información en el diseño y la ejecución de edificios.

- Nuevas tecnologías de construcción.

- Técnica para evaluación, mantenimiento y reparación de edificios.

- Desarrollo en el diseño de instalaciones.

He aquí, pués, la panorámica de lo que se juzga prioritario en el campo de la investigación referente al sector edificación, al máximo nivel científico y técnico oficial dentro del marco de la CEE.

Pero hay que destacar otro aspecto importante para que este esfuerzo común pueda realizar la investigación científico-técnica en áreas de un progreso que sea realmente eficaz; $y$ este aspecto se refiere a la necesidad de una investigación prenormativa.

La Investigación Prenormativa puede definirse como una investigación que suministra las bases científicas y tecnológicas para el establecimiento, perfeccionamiento y cumplimiento de reglamentaciones y normas. Se considera un medio importante para crear el Mercado Europeo integrado, promocionando la competitividad de la Industria Europea, y proteger al consumidor y al medio ambiente.

Como puede leerse en los documentos emanados en la CEE (III/D-2), desde hace un quinquenio, el establecimiento de la reglamentación y la normativa se ha considerado como una necesidad para la consecución del mercado interior. La presión ejercida por el merca. do sobre la Industria, los Institutos nacionales de Normalización, y por la Comisión en su empeño de pro. ducir directivas y reglamentaciones y normas deriva. das de aquéllas ha sido tal, que se ha realizado una enorme cantidad de trabajo por los organismos euro. peos de normalización, tales como el CEN (Comité Europeo de Normalización), el CENELEC (Centro Europeo de Normalización Electrónica), etc. Y todavia queda muchisimo por hacer; de ahi que exista un conven. cimiento de la necesidad de estudiar una reorganiza. ción de las Normas Europeas y de lograr una mayor

http://informesdelaconstruccion.revistas.csic.es 


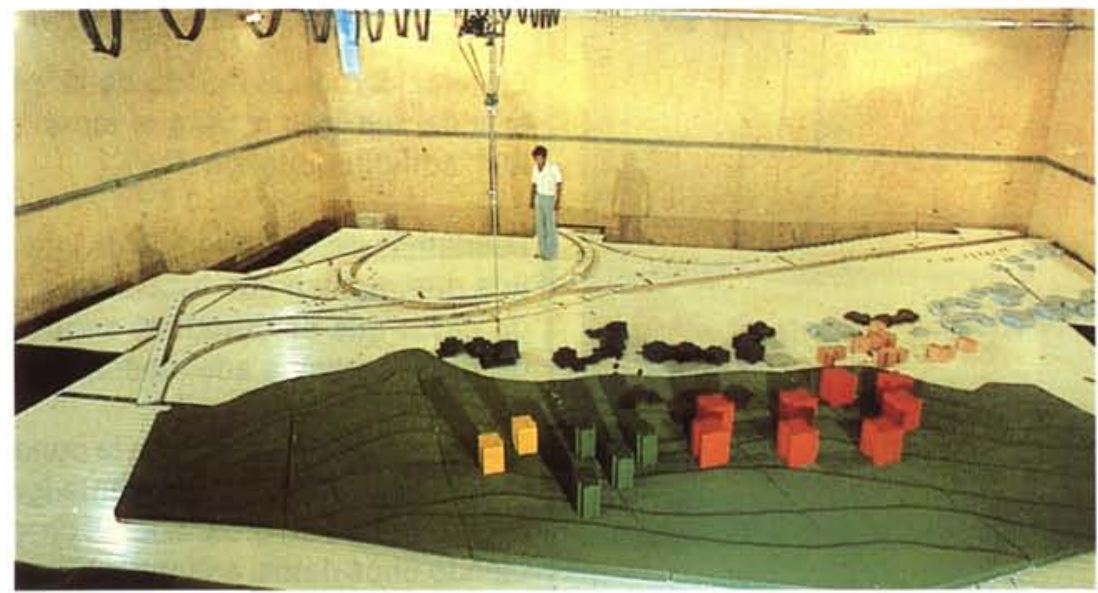

Fig. 2.-Estudios acústicos sobre maquetas realizadas en el CSTB de Francia.

coordinación en los programas de Investigación y Desarrollo.

A este fin, conviene señalar la existencia del IRDAC (Industrial R \& D Advisory Committee of the Commission of the European Communities), organismo llamado a desempeñar un importante papel en la investigación prenormativa.

La Investigación Prenormativa deberá ser de tal índo. le que proporcione una base científica y tecnológica para la preparación y posterior desarrollo de instrumentos legales tales como Directivas. Estos instrumentos son propuestos generalmente por los Poderes Públicos para el interés y el bienestar de las gentes. Por lo tanto, deberán referirse a cuestiones tales como: medio ambiente, biotecnologia, salud médica, etc.

Como observa J. Rilling, Director científico del CSTB y coordinador del grupo anteriormente citado del ENBRI, al redactar el documento de introducción al Sim. posio de Luxemburgo, nos hallamos en la era de la protección ambiental.

El reciente incremento del interés a escala mundial del efecto invernadero, del agotamiento de la capa de ozono y de la lluvia ácida, arrojará indudablemente nuevos conocimientos sobre la contribución directa 0 indirecta de $\mathrm{CO}_{2}, \mathrm{NO}_{x}, \mathrm{CH}_{4}, \mathrm{CFC}$ y $\mathrm{SO}_{2}$ en los edificios. Y, como advertía la profesora Otero Pastor en el editorial de un reciente número de nuestra revista dedicada a la conservación del medio ambiente, en el plano técnico, "la propia dinámica de la preocupación por la Naturaleza, ha ido desplazando los centros de interés desde los estudios de alcance territorial extenso o muy extenso, a la concreción de acciones en proyectos más definidos y particularizados, entre los que se encuentran todos aquéllos relativos a la restauración del paisaje. La búsqueda del planteamiento más acer- tado induce asi a ingenieros, arquitectos y otros profesionales, un nuevo modo de ver las cosas, mejorando las técnicas y buscando los materiales - las plantasmás apropiadas para ello dentro de este nuevo enfoque; restituyendo, en definitiva, todos aquellos espacios que puedan verse afectados por las distintas obras y construcciones".

En cuanto a la investigación referente al ruido, se han hecho grandes programas mediante gráficos de computadores para simular y calcular el nivel de ruido en ciudades situadas al borde de fuentes de ruido tales como autopistas o vías férreas, con objeto de conseguir análisis sistemáticos sobre el impacto en los alrededores de una autopista urbana.

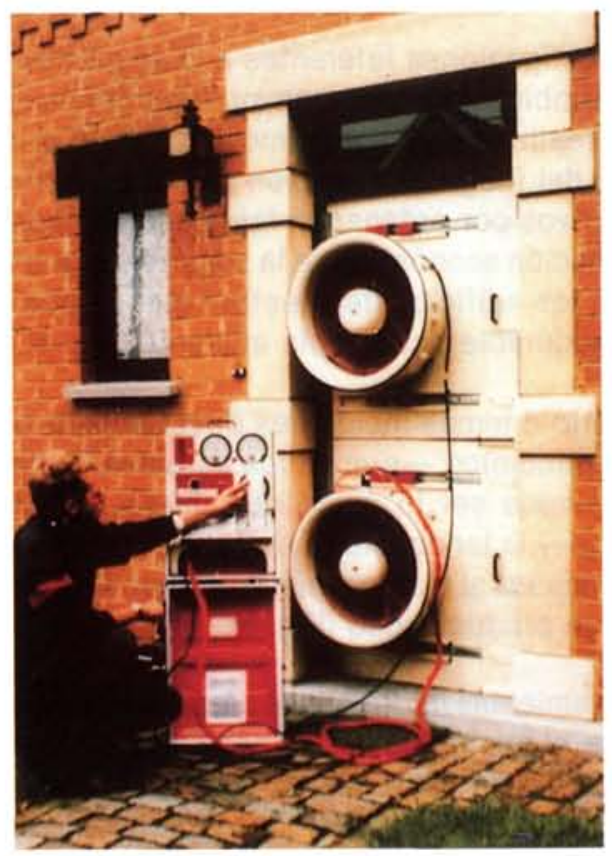

Fig. 3.-Determinación de la estanquidad al aire de una habitación. Ensayo realizado en el CSTB de Bélgica.

http://informesdelaconstruccion.revistas.csic.es 


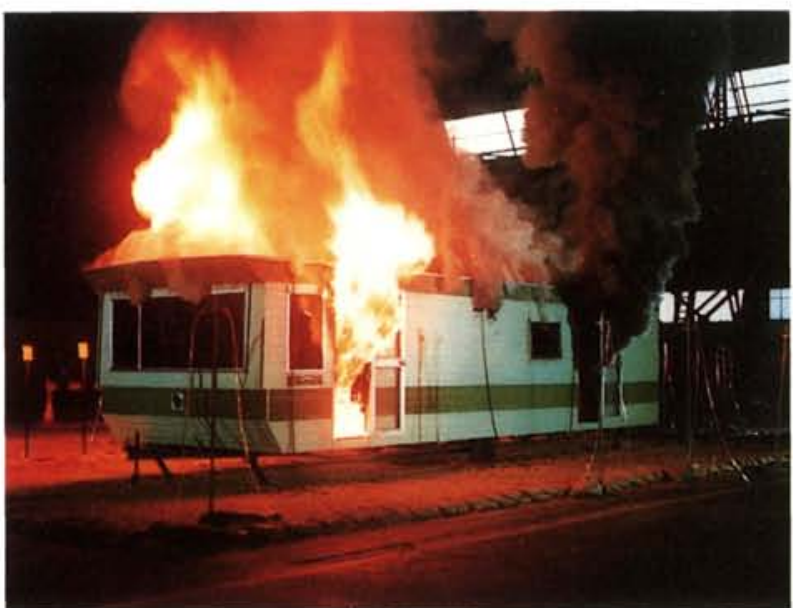

Fig. 4.-Ensayo de resistencia contra el fuego realizado por el BRE de Inglaterra.

Lo importante es definir unas condiciones de salud, incluyendo por ejemplo el ruido (Fig. 2) y la calidad del aire. O saber cuál es el nivel de concentración de $\mathrm{CO}_{2}$ en el aire interior o el de radón aceptables. Muchas de estas cuestiones poseen estrecha conexión con las propiedades de los materiales; y se necesita un conjunto mejor de técnicas de medida para determinar la emisión de agentes polucionantes en los materiales y la concentración de gases específicos en los edificios. (V. gr. el problema del radón). También existe una gran conexión con las instalaciones. Las concentraciones en los elementos polucionantes están relacionadas con la ventilación y los sistemas de diseño de los edificios, por lo cual necesitamos mejores estrategias, un mejor diseño y control de la ventilación (Fig. 3).

Las investigaciones referentes a la seguridad ante el fuego también adquieren mayor importancia. Los progresos realizados en los últimos quince años sobre la ciencia del fuego, modelación y control de los sistemas activos por ordenador, las redes estocásticas y la simulación económica de la seguridad contra incendios en los edificios demuestran que es posible dar un considerable paso hacia adelante (Fig. 4).

En cuanto a temas incidentes directamente en el aspecto económico -que en los actuales tiempos hay veces parece ser lo único que interesa en nuestra sociedad - la tendencia es potenciar las investigaciones enfocadas al mantenimiento y rehabilitación en términos de productividad (Fig. 5).

Las herramientas informáticas presentan, más frecuentemente, el mundo de la Edificación. Una vez establecidas, para asegurar la gestión empresarial, comienzan a imponerse en la fase de concepción de los proyectos y en la organización de las obras, de tal forma que hace posible la unión entre las actitudes de concep. ción y fabricación, mediante la ayuda de los ordena. dores (sistemas CAD y CAM).

En relación con el mundo de la informática y la electrónica, también resalta el tema "punta" y actual de los "edificios inteligentes".

Podríamos definir los edificios inteligentes como aquellos que regulan sus funciones a través de una toma de datos que se introduce en un ordenador, provisto de los programas adecuados para su gobierno.

Como señala Florez Alía en la ponencia presentada en Ia Jornadas sobre Edificios Inteligentes " "estamos en la generación de ordenadores programados que, recibiendo unos datos, aplican a ellos sus programas y resuelven las situaciones posibles y previstas. Pero desde hace años se trabaja en ordenadores llamados de inteligencia artificial, que serán capaces de actuar en una situación imprevista, generando programas de resolución de la misma, a partir de su software. Es decir, ordenadores dotados de una programación capaz de generar programas para situaciones no previstas. Este es el futuro, por lo que resulta interesantísima la investigación en esa dirección".

Por otra parte*, «el desarrollo experimentado por la electrónica en las dos últimas décadas, tanto en el aspecto tecnológico y de fiabilidad, como en el de "estandarización" y abaratamiento de producto de medio, alto y muy alto grado de integración, ha permitido la invasión de las tecnologias electrónicas en multitud de campos que han supuesto una verdadera revolución en el control de procesos, la informática y las comunicaciones. La fiabilidad y versatilidad del control electrónico han ido sobreponiéndose a los sistemas independientes y por tanto de control distribuido sin interacción entre ellos".

Por último, no queremos dejar de referirnos a la domótica (domus, casa) sugestivo tema para un próximo futuro. Ya hace años, en la revista "Informes de la Construcción ${ }^{* *}$, dábamos unas ideas sobre las importantes modificaciones que habria de sufrir la distribución de las superficies de los espacios en los edificios futuros. Concretándonos a la vivienda, la transforma. ción del cuarto de estar en un "hogar electrónico" tenia que facilitar un acceso colectivo a los equipos que alli se han de concentrar. La interpretación del vídeo y de la cadena $\mathrm{Hi}$-Fi, el desarrollo del videoproyector doméstico y, posteriormente, de la pantalla de gran.

\footnotetext{
- Cotec 90 - Valencia - Noviembre 1990.

- J. Aguinaga - Citada Ponencia.

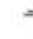

“ F. Aguirre de Yraola: "EUROBILD y el futuro de la construcción" "Informes de la Construcción", n. 363, 1984.
} 


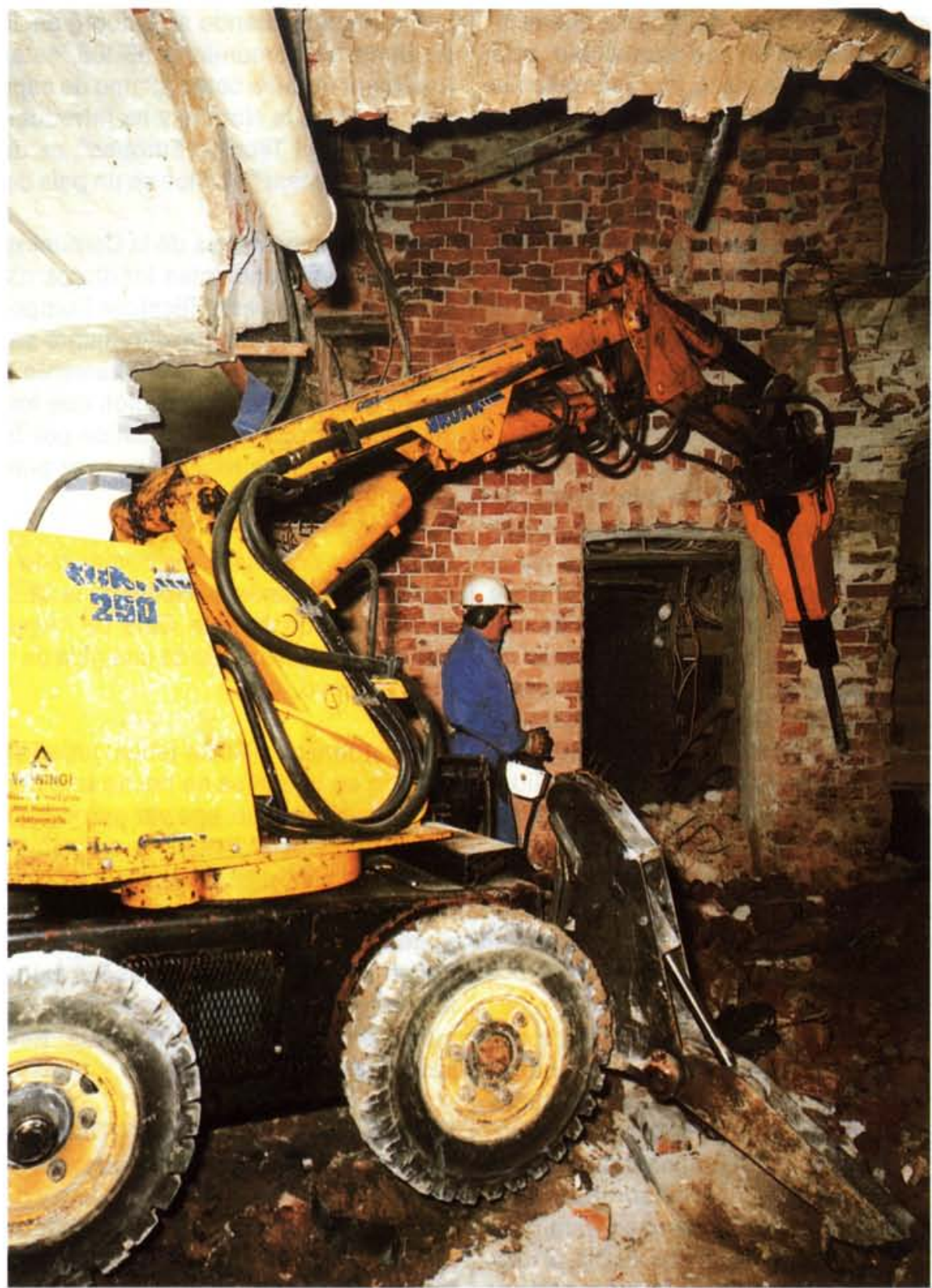

Fig. 5.- Modernas tecnologias de rehabilitación, por control remoto, realizadas en Finlandia.

des dimensiones combinada con la penetración de la imagen de alta definición, deberán constituir las principales etapas para la creación de estos espacios, en los que la perspectiva necesaria para la contemplación del muro o pared-pantalla exigirá una distancia minima de tres veces su altura.

Los estudios del IDATE se han extendido sobre las correlaciones posibles entre los tres modelos diferentes que configurarán la realidad socio-económica del mañana, en función de los nuevos equipos electrónicos, tanto domésticos como de oficina, talleres, centros de enseñanza, etc., y que determinarán una morfologia de los espacios habitables. ¿Cuál será la importancia futura del trabajo a domicilio? El tele-trabajo, la tele- enseñanza, la tele-financiación... modificarán profundamente la vida actual y podrán incluso producir una considerable reducción en el flujo del transporte.

La tercera Revolución industrial será fuente de nuevos equipos industriales y de una remodelación de las infraestructuras, y de los equipos, en particular, en el área de la vivienda.

Como ejemplo de las iniciativas que van tomando, afortunadamente, los Poderes Públicos en el ámbito de la Investigación, el Banco Hipotecario de España ha de. cidio acometer la empresa de construir lamejor de las viviendas imaginable dados el estado de la cultura y de las tecnologias a escala mundial en este sorpren- 
dente final del siglo XX. El Hogar del Futuro, que el citado Banco estatal presenta en esa exposición, será construido realmente en breve plazo, incorporando las innovaciones conceptuales y tecnológicas que ahora tan sólo se sugieren.

Para finalizar estas líneas, después de esta panorámica sobre las tendencias de la actividad y de la investigación en materia de edificación, damos unos datos que juzgamos muy interesantes como orientación de la actual situación de España en el contexto de la prenormativa europea, base sin duda para un progreso realmente eficaz del sector.

En 1960 se creó la UEAtc (Union Europeénne por l'Agrément Technique dans la Construction) que agrupa, en la actualidad, a 14 Institutos de otros tantos paises europeos, entre ellos el Instituto de Ciencias de la Construcción Eduardo Torroja de España, y que tiene por finalidad facilitar y estudiar la evaluación de materiales, procedimientos y sistemas constructivos no tradicionales o que implican nuevas tecnologías, tanto a través de Directrices o Guías Técnicas como a través de programas de investigación generados en cada Instituto miembro, así como la de convalidar en el país receptor los ensayos hechos en el país exportador por el Instituto miembro correspondiente. En España el "Agrément" es conocido como Documento de Idoneidad Técnica (DIT), y el ICCET ha sido el Instituto miembro de la UEAtc durante todos es. tos años.

Esta forma de proceder, iniciada antes de la propia existencia de la CEE, ha permitido disminuir las barreras técnicas existentes entre los paises con respecto a materiales o procedimientos no tradicionales, asi como poner a disposición de los técnicos unas Directivas (hoy Guías) de uso que les permiten utilizar los mismos materiales en la construcción con garantías de seguridad, habitabilidad y durabilidad. EI DIT, al mismo tiempo, favorece el desarrollo de la innovación y puede considerarse como una primera etapa dentro del proceso de normalización, reglamentación y certificación.

La CEE ha seguido atentamente los trabajos de la UEAtc desde 1975 y ha manifestado su interés por la marcha del Agrément, como una de las vias de armonización técnica y de apertura a la innovación de los productos de construcción sobre el plano europeo y, naturalmente, cuando se elaboró en 1986 el proyecto de Directiva comunitaria $89 / 106$, ésta ha incluido el Agrément técnico como $2^{\circ}$ tipo de especificación técnico (el $1^{\circ}$ es la Norma) y ha introducido el concepto de "Agrément Técnico Europeo", es decir, la emisión de un "Agrément" técnico en un país de la Comunidad.

Los Estados-miembros de la Comunidad han designado sus correspondientes institutos nacionales* para emitir los "Agréments Técnicos Europeos", y estos institutos se integrarán próximamente en el seno de una organización prevista explícitamente por la Directiva de Productos de Construcción que tomará el nombre de EOTA (European Organization por Technical Approvals), habiéndose reunido por vez primera en febrero de 1990.

Este nuevo organismo ha comenzado por preparar un proyecto de mandato-cuadro de la CEE a la EOTA para la elaboración de una Guía de "Agrément" técnico europeo, y el plano-tipo de una guía de "Agrément" técnico europeo.

Y en octubre de 1990 ha tenido lugar, en Bruselas, una reunión en la que se ha hecho una declaración de intenciones para que, una vez aprobados los Estatutos, se constituya próximamente de un modo oficial dicha EOTA. Los Institutos trabajan ya en las Guías de "Agrément" Técnicas mandadas por la CEE ${ }^{\star \star}$ y los "Agréments" Técnicos Europeos sobre los productos de construcción para los cuales las exigencias esenciales previstas por la Directiva de Productos de Construcción se consideran importantes. Estos trabajos se reconocen como prioritarios y urgentes.

Resulta pues, que la puesta en obra operativa del "Agrément" Técnico Europeo reglamentario exige de manera general que: la EOTA exista y funcione; que los Estados hayan transpuesto la Directiva 89/106 en su legislación nacional; y que los Documentos Interpretativos relativos a las exigencias esenciales se hayan establecido y aprobado, así como las Normas fundamentales armonizadas, avalando los Documentos In. terpretativos.

Para todo esto será preciso un cierto tiempo. Por lo cual, la UEAtc ${ }^{\star \star *}$ ha decidido proseguir sus trabajos actuales (establecimiento de Guías de "Agrément" Técnicas nacionales) durante el tiempo necesario para pasar, en las mejores condiciones posibles, de la solución

\footnotetext{
* El Estado español ha designado al Instituto de Ciencias de la Construcción Eduardo Torroja como "Organismo Especial autorizado para la concesión de los Documentos de Idoneidad Técnicos Europeos".

* Conviene advertir que, en marzo de 1990, se incorporaron a la UEAtc Finlandia, Noruega y Suecia.

*** Un ejemplo de estas Guias puede verse en el n. 410 de esta Revista y en la Monografía n. 403 , también del ICCET, sobre: "Guía Técnica UEAtc para la evaluación de colas para revestimientos cerámicos".
} 
transitoria (disposiciones nacionales) a la solución europea armonizada.

Por otra parte, es de destacar también la integración del ICCET en el organismo internacional "IDEA" (Innovation Development European Assessment), o Diagnóstico de Innovación Europeo, documento que constituye una ayuda para la decisión, destinado a las empresas e industriales de la construcción que desean lanzar un nuevo producto o procedimiento en España y/o sobre los mercados de uno o varios países que integran la CEE.

Como el costo del proyecto depende de la adaptación del producto o procedimiento a la clientela potencial, y de la posición técnica y económica del producto o procedimiento en relación a sus concurrentes, un buen proyecto debe, pues, basarse en la juiciosa elección de las opciones técnicas y de las acciones para concretarlas.
El Diagnóstico de Innovación Europeo aprecia las ventajas y evalúa los puntos débiles y los riesgos del proyecto, en cada uno de los paises destinatarios; indica las mejoras y modificaciones técnicas a realizar eventualmente y las etapas claves de su realización.

Esta actividad está auspiciada por el Programa SPRINT de la CEE, que consiste en el desarrollo transnacio. nal de la infraestructura de ayuda a la innovación y a la transferencia de las tecnologías.

En la actualidad, integran el programa varios de los centros de investigación de la construcción más competentes de Europa, y Grecia es el único pais de la CEE que todavía no se ha integrado, aunque se espera lo hará en fecha próxima.

Confiamos en que estas informaciones sean de utilidad para el sector, teniendo en cuenta el año 1993, por razones obvias.

\section{última monografía del ICCET/CSIC (n. $\left.{ }^{\circ} 403\right)$}

\section{GUÍA TÉCNICA UEATC PARA LA EVALUACIÓN DE COLAS PARA REVESTIMIENTOS CERÁMICOS}

Traducción y adaptación: CÉSAR DEL OLMO

Dr. en Ciencias Químicas

Madrid, diciembre 1990

La presente monografía contiene los criterios adoptados por los Institutos miembros de la UEAtc para poder apreciar la idoneidad técnica de las colas que se emplean para la colocación de revestimientos cerámicos sobre los distintos elementos constructivos en que normalmente se aplican (muros interiores y exteriores y suelos interiores y exteriores).

El documento se divide en los siete capítulos siguientes: El primero contiene una breve introducción al tema. En el Capítulo segundo se enumeran las reglas generales de calidad de estos productos. El Capítulo tercero incluye la terminología y definiciones de los productos de encolado así como de sus características de puesta en obra. El Capítulo cuarto define los métodos de ensayo adoptados para la medida de las características técnicas. El quinto contiene las especificaciones mínimas que deben satisfacer. El Capítulo sexto se refiere al control de calidad mínimo que debe adoptarse en la fabricación de estos materiales. Y el séptimo y último contiene la información técnica que deberá figurar en cada Documento de ldoneidad Técnica. 